\title{
La industria farmacéutica y la sostenibilidad de los sistemas de salud en países desarrollados y América Latina
}

\author{
The pharmaceutical industry and the sustainability \\ of healthcare systems in developed countries and in Latin America
}

Antonio Iñesta ${ }^{1}$

Luis Angel Oteo ${ }^{2}$

\footnotetext{
${ }^{1}$ Área de Análisis del U so de los M edicamentos, Escuela Nacional deSanidad, Instituto deSalud Carlos III, Ministerio de Cienciae Innovación. C/Sinesio Delgado 8. 28029 M adrid España. ainesta@isciii.es

${ }^{2}$ Departamento de Desarrollo Directivo y Gestión de Servicios Sanitarios, Escuela Nacional deSanidad, Instituto de Salud Carlos III, M inisterio deCienciaelnnovación, España.
}

\begin{abstract}
The global economic crisis and its impact on public finances in most developed countries are giving rise to cost-containment policies in healthcare systems. Prevailing legislation on medication requires the safety, quality, and efficacy of these products. A few countries include efficiency criteria, primarily for new medication that they wish to include in public financing. The appropriate use of generic and "biosimilar medication" is very important for maintaining the financial equilibrium of the $\mathrm{H}$ ealth Services. The problem in Latin America is that not all multisource products are bioequivalent and not all countries have the resources to conduct bioequivalence studies in vivo. The European $M$ edicines A gency in 2005 adopted guidelines on "biosimilar medicines" and thirteen of them were subsequently approved for general rel ease. Benchmarking of this model by other countries would be important. The influence of the pharmaceutical industry on political and administrative areas is enormous and control is necessary. The pharmaceutical companies claim that they act with corporatesocial responsibility, therefore, they must ensure this responsibility toward society. Key words Drug industry, Drug regulations, Sustainable development, Latin America, D eveloped countries

Resumen La crisis económica y su impacto en las finanzas públicas en la mayoría de los países desarrollados, están originando políticas de contención del gasto en los servicios de salud. Las leyes actuales del medicamento exigen calidad, seguridad y eficacia deestos productos. Algunos países incluyen criterios de eficiencia para los nuevos medicamentos que desean ser incluidos en la financiación pública. El consumo apropiado de genéricos y "medicamentos biosimilares" es muy importante para mantener el equilibrio financiero de los servicios de salud. El problema en América Latina es que no todos los productos multifuentes son bioequivalentes y no todos los países tienen los recursos necesarios para realizar los estudios de bioequivalencia in vivo. La Agencia Europea del M edicamento en 2005 aprobó la directriz sobre "medicamentos biosimilares" y después se han autorizado trece de ellos para su comercialización. El referenciamiento de este modelo por otros países seria importante. La influencia de la industria farmacéutica sobre las áreas políticas y administrativas es necesario controlarla. Las compañías farmacéuticas afirman que actúan con responsabilidad social corporativa, por ello, se debería garantizar el cumplimiento de la misma con la sociedad.

Palabras clave Industria farmacéutica, Regulación de medicamentos, Desarrollo sostenible, América Latina, Países desarrollados
\end{abstract}




\section{Introducción}

El sector de la salud forma parte esencial de la economía productiva y social de los países avanzados, y es generador continuado de riqueza, prosperidad y empleabilidad cualificada. Su alta interdependencia con otros mercados estratégicosy gl obales, convierten a su cadena de diseño y de valor en un cluster de innovación disruptiva deprimer orden. En losúltimos 40 años esta economía del bienestar ha sido el sector con más rápido crecimiento en Europa y Estados Unidos, representando hoy un volumen denegocio superior a los 3,5 billones de euros ${ }^{1}$.

La industria de la salud está presente en todas las formas y procesos de globalización, y sus mercados de bienes, manufacturas y servicios, así como susfactores de producción se han mundializado en las últimas décadas de manera vertiginosa². La inversión en I +D (Investigación y Desarrollo) en productos farmacéuticos y biotecnología no se ha resentido de forma significativa a pesar de la crisis económica, en parte debido a los procesos de crecimiento orgánico de las grandes compañías a través de adquisiciones y fusiones ${ }^{3}$.

El insumo farmacéutico representa en términos relativos más del $50 \%$ de los activos que se generan en la "industria de la salud", y su crecimiento - a pesar de la crisis de innovación- sigue siendo abrumador en volumen de ventas y resultados económicos4.

La crisis financiera global y sus efectos en la economía de las empresas no han representado en el sector farmacéutico cambios significativos en su rentabilidad. Además, sus ratios de beneficio sobre recursos propios siguen siendo elevados y sostenibles en comparación con otros sectores estratégicos de la economía global ${ }^{5}$.

El propósito de esteartículo es analizar el insumo farmacéutico dentro del sector salud como parte de la economía productiva y social de los países, en el contexto deAmerica Latina, la Unión Europea y otros países desarrollados, en un momento en que la crisis económica está planteando políticas de contención del gasto sanitario. Entre estas políticas se consideran: la inclusión de criterios de eficiencia para los nuevos medicamentos que desean ser incluidos en la financiación pública, la promoción del consumo de medicamentos genéricos y "medicamentos biosimilares", el impulso de la competencia y liberalización de determinadas actividades de la cadena de valor del sector, así como la extensión de la responsabilidad corporativa de las compañías farmacéuticas y su compromiso social.
Para ello en primer lugar, trataremos del difícil equilibrio entre una industria con tendencia a originar fuertes incrementos en la factura farmacéutica financiada públicamente y un sistema público obligado a la contención del gasto; en segundo lugar, se incluyen algunas de las medidas básicas de ajuste del gasto farmacéutico para poder asumir las innovaciones; se sigue con la exigencia de una mayor regulación de la industria farmacéutica, unida a la crisis de racionalidad en el uso de los medicamentos y en la investigación biomédica; y por último se reflexiona sobre la responsabilidad social y el buen gobierno en el sector farmacéutico.

\section{La industria farmacéutica y su impacto en las finanzas públicas}

El proceso de capitalización creciente de esta industria, se debe fundamentalmente a una conjunción de factores como: la consolidación de los activos fijos a largo plazo; las infranqueables barreras de entrada; la consistencia competitiva de sus estrategias globales; el continuado beneficio operativo sobreventas einversión; así como la poderosa capacidad de influencia sobre los decisores del sector y su eficiente red tentacular que llega a todos los ámbitos y niveles de actuación sanitaria, integrando virtualmente a los agentes internos en su propia cadena de valor y controlando las redes sociales y profesionales adyacentes ${ }^{6}$.

El tejido empresarial debasetecnológica dela biomedicina y de la salud humana, es hoy uno de los enfoques más visibles del desarrollo económico y social de las economías modernas? ${ }^{7}$ Sin duda, estesector de la economía social precisa de regulación tanto nacional como internacional, de políticas de precios transparentes y socialmente responsables, de garantías públicas para blindar la propiedad industrial y de un sistema de innovación como política de los gobiernos para impulsar las funciones más estratégicas y de mayor valor añadido de esta industria ${ }^{8}$.

Bien es cierto también que la industria farmacéutica tiene sus propias incertidumbres estructurales y de mercado, derivadas de la caducidad de los derechos de propiedad de determinados fármacos líderes en ventas, la incierta cartera de insumos en proceso de investigación (atasco en los procesos de innovación) con una tenue productividad, el incremento del uso defármacos biosimilares, la presión a la baja del precio de los medicamentos biológicos o los potenciales ajus- 
tes estructurales como consecuencia de las futuras concentraciones o megafusiones en ciernes.

Pero delaciencia y la técnica dependen buena parte de las soluciones a las necesidades de salud de las personas y poblaciones, y de cómo se promueva y gestione la innovación disruptiva dependerá la creación de nuevos mercados farmacéuticos emergentes competitivos?. Todos estos cambios que buscan una mayor competitividad y valor añadido en los procesos de innovación biomédica, están generando un nuevo modelo denegocio adaptando estructuras, competencias y dinámicas de colaboración con todos los agentes que están integrados en la cadena de valor del sector sanitario (Figura 1).

El interés social exigeuna mayor cooperación de las empresas farmacéuticas con los gobiernos y autoridades reguladoras para mejorar la calidad de la evaluación y garantizar en consecuencia más altos estándares de seguridad, eficacia y eficiencia en los medicamentos. Además, la crisis económica y su impacto en las finanzas públicas en la gran mayoría de los países occidentales con arquitecturas sociales avanzadas, está planteando políticas de contención del gasto sanitario que exigen racionalidad y buen gobierno.

\section{La regulación de los medicamentos y la necesidad de eficiencia}

Uno de los objetivos básicos de una política de medicamentos es asegurar que están disponibles medicamentos seguros, eficaces y decalidad, para cubrir las necesidades sanitarias de un país. Además de los requerimientos anteriores algunos países (Cuadro 1) también consideran la eficiencia, es decir la relación coste/beneficio antes dela introducción de un nuevo medicamento.
El desarrollo deesta política, precisa considerar al medicamento como un bien social que debe estar disponible en el sistema sanitario para mejorar la salud de los ciudadanos, que el acceso al mismo debeser universal y equitativo, y que debe ser usado de forma eficiente de acuerdo con la evidenciacientífica disponible.

Los objetivos generales de una política farmacéutica nacional son asegurar el acceso a los medicamentos, la calidad (calidad, inocuidad y eficacia de todos los medicamentos) y el uso racional de los mismos ${ }^{10}$.

En el mundo, las leyes del medicamento de primera generación, hasta los años sesenta, exigían calidad y seguridad pero no eficacia de los medicamentos; pero como consecuencia de la catástrofe de la talidomida en el año 1962, las de segunda generación exigían calidad, seguridad y eficacia demostrada a través de ensayos clínicos controlados. Las de tercera generación además de los requisitos anteriores incluyen los criterios de eficiencia y las condiciones para el uso racional de los medicamentos.

Actualmente son varios los países que han ido incorporando la evaluación económica delos nuevos medicamentos como una ayuda a la toma de decisiones. Considerada como la "cuarta garantía", la evaluación farmacoeconómica pretende contribuir al uso eficiente de los recursos disponibles en el ámbito sanitario. Australia y $\mathrm{Ca}$ nadá fueron pioneros en los años noventa al incorporar el análisis coste- efectividad en la toma de decisiones para incorporar los nuevos medicamentos en la financiación pública ${ }^{11}$.

Varias administraciones sanitarias, han publicado guías de evaluación económica con vistas a la autorización de nuevos medicamentos, fijación de precioso financiación pública. En Australia, Canadá e Israel la autorización de nuevos

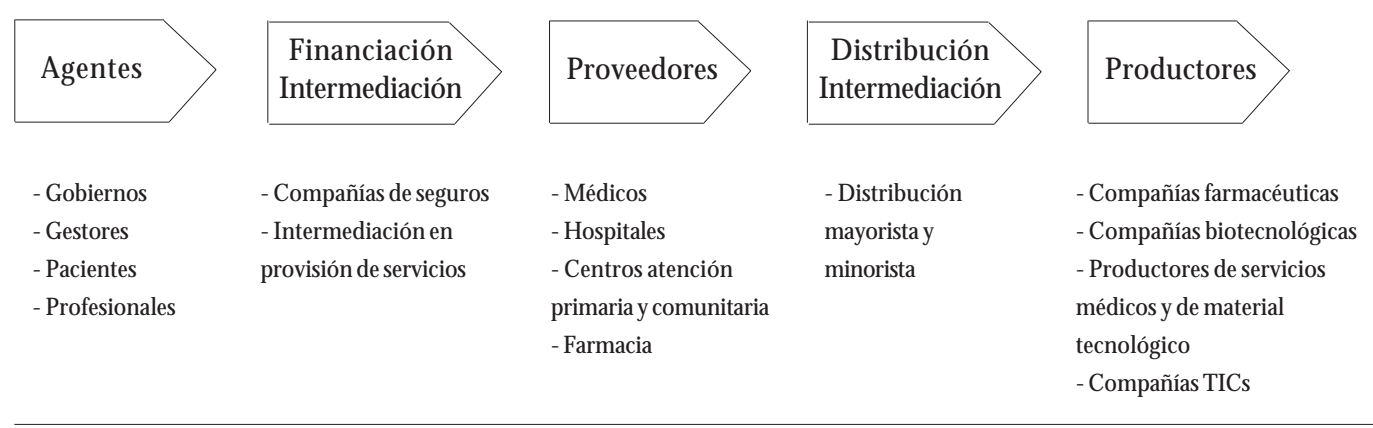

Figura 1. Cadena de valor del sector sanitario. 
Cuadro 1. Experiencias en guías de evaluación que se remiten para inclusión de medicamentos en la financiación pública, bien exigidas o recomendadas. Últimas versiones disponibles en los países identificados.

\begin{tabular}{|c|c|}
\hline 2001 & $\begin{array}{l}\text { Inglaterra y Gales, NICE, Directrices para fabricantes y promotores, julio } 2001 \text { http:// } \\
\text { www.nice.org.uk/niceM edia/pdf/technical guidanceformanufacturersandsponsors.pdf }\end{array}$ \\
\hline 2002 & $\begin{array}{l}\text { Israel, guía para solicitar la inclusión de un medicamento en la financiación pública, enero } \\
\text { 2010, versión no } 8 \text { www.health.gov.il/download/forms/a3716_aclala_Eng2010.doc }\end{array}$ \\
\hline 2002 & $\begin{array}{l}\text { Bélgica, guía recomendada para inclusión de un medicamento en la financiación pública, } \\
2006 \text { http://www.kce.fgov.be/D ownload.aspx?l D =493 }\end{array}$ \\
\hline 2006 & Canadá, 3a edición http://www.cadth.ca/media/pdf/186_EconomicGuidelines_e.pdf \\
\hline 2003 & Taiwan http://www.ispor.org/PEguidelines/source/2006_PE_Guidelines.pdf \\
\hline 2008 & $\begin{array}{l}\text { Australia, version } 4.3 \text { diciembre } 2008 \\
\text { http://www.pbs.gov.au/html/pdf/industry/H ow_to_list_on_the_PBS/ } \\
\text { Elements_of_the_listing_process/PBAC_guidelines/PBAC4.3.2 }\end{array}$ \\
\hline 2009 & $\begin{array}{l}\text { EUA, versión } 3.0 \text { octubre } 2009 \text { guía para inclusión en formulario http://www.fmcpnet.org/ } \\
\text { index.cfm?c=news.details\&a=wn\&id=F27AD5FE }\end{array}$ \\
\hline
\end{tabular}

medicamentos requiere la presentación de estudios de evaluación económica. En el sitio Web de Ia ISPOR, "Pharmacoeconomic Guidelines Around the World"12, se incluyen 35 directrices sobre evaluación económica de 27 países, queincluyen 26 directrices farmacoeconómicas, 8 Directrices de remisión para la Lista de Formulario y 1 para publicación en una revista (Cuadro 1 ).

Una encuesta realizada en el estudio de VigiIancia y Evaluación de Política Farmacéutica Nacional, conducido por la Organización Panamericana de la Salud y sistematizada por el Núcleo de Assistência Farmacêutica/Escola Nacional de Saúde Pública de Brasil, a 40 países de América, aunque con datos de 2005-2006 que han sido superados, es indicativa de que se van aplicando las políticas y regulación de medicamentos en America Latina $(A L)$. Delos 40 países respondieron 26 y de ellos 5 no disponían aún de un marco regulatorio para el sector ${ }^{13}$.

Un aspecto importante a considerar es el de los medicamentos genéricos. Los genéricos son medicamentos que se comercializan cuando termina el periodo de patente de los medicamentos de marca, son más baratosy tienen igual calidad, seguridad y eficacia quelas marcas de referencia.

El consumo de genéricos es muy importante para mantener el equilibrio financiero delosservicios de salud o de seguridad social y para reducir lo gastado directamente por los ciudadanos. Como sesabe la prescripción degenéricostienen un componente educativo (acceso a información no sesgada) relacionado con la educación recibi- da por los profesionales sanitarios en las facultades, y otro componente económico (hacer sitio en los presupuestos de los servicios de salud para que puedan entrar los nuevos medicamentos que van a llenar vacíos en la terapéutica) ${ }^{14}$.

Losmedicamentos genéricosson una importante herramienta para mejorar el acceso a los medicamentos porque constituyen la principal forma de competencia del mercado farmacéutico, lo que se traduce en reducción de precios e incremento del beneficio social. Las políticas de genéricos contemplan la promoción eincentivos a la producción y uso de genéricos a través de diversos mecanismos, incorporados en las diferentes etapas del proceso de abastecimiento de medicamentos: producción, registro, dispensación, prescripción y uso.

En la Unión Europea (UE) cuando se habla de genéricos se refiere a medicamentos que se nombran por su Denominación Común Internacional $(\mathrm{DCl})$, aunque puedan ir acompañados por el nombre del laboratorio fabricante 0 garante, que ha demostrado su bioequivalencia en ensayos in vivo con la marca de referencia y que son en todo intercambiables. La nomenclatura en $A L$ es diferente y se podría equivaler al de producto farmacéutico multifuente terapéuticamente equivalentey por tanto intercambiablecon la marca de referencia, simplificando se va a llamar producto multifuente bioequivalente.

El problema en $A L$, es que no todos los productos multifuentes son bioequival entes y no todos los países tienen los recursos necesarios para 
realizar los estudios de bioequivalencia in vivo. De acuerdo con un trabajo comparativo de los requerimientos delos estudios debioequivalencia (estudios farmacocinéticos in vivo en humanos) entre los Estados Unidos de América (EUA), Canadá y sietepaíses deAL (Argentina, Brasil, Chile, Costa Rica, Cuba, M éxico y Venezuela) ${ }^{15}$, con información disponible a julio de 2006:

- De los 98 principios activos analizados, solamente a 5 de ellos se les requieren estudios de bioequivalencia en los 9 países estudiados (ácido valproico, carbamazepina, ciclosporina, fenitoína y verapamilo).

- Los países con mayor número de principios activos a los que se les exigen estudios de bioequivalencia son Canadá (92) y EUA (87).

- En los países de AL estudiados se han encontrado los siguientes resultados (número de principios activos del Listado a los que se les exigen estudios de bioequivalencia: Brasil (89); M éxico (59); Venezuela (21); Chile(15); Argentina (15); Cuba (12) y Costa Rica (8).

- Se observó una similaridad entre países de exigencia de estudios de bioequivalencia respecto a principios activos de alto riesgo sanitario, lo que brinda un sólido fundamento a tomar este criterio de riesgo en el momento de tomar decisiones sobre este tipo de exigencias.

Aunque es necesario poner al día el estudio, este análisis comparativo demuestra la diversidad de las realidades regulatorias en cada uno de los países analizados.

Otro capitulo importante por su importancia económicay deasequibilidad esel delos"medicamentos biosimilares" ("productos medicinales biológicos similares"), debido a la complejidad de muchos de estos productos y de los costes implicados en desarrollar las versiones biosimilares, se estima que solo un número reducido de compañías de genéricos proveerán estos productos.

La directriz sobre "medicamentos biosimilares" fue adoptada por el Comité para Medicamentos para Humanos (CH M P) de la Agencia Europea del M edicamento (EM A) en septiembre de 2005 y abrió la puerta para su comercialización en la UE desde el punto de vista legislativo.

H asta la fecha, en la UE se han otorgado 13 autorizaciones decomercialización debiosimilares basadas en seis desarrollos. Se han rechazado o suprimido 3 desarrollos lo quesignifica un $67 \%$ detasa de éxito, por tanto de confianza en el sistema regulatorio de la UE. En Alemania, se estableceel mismo grupo deprecios de referencia para todas la somatropinas (incluyendo biosimilares). La "filosofía biosimilar" de la UE supone tam- bién una guía para el desarrollo de la misma en otros muchos países (somatropina, epoetina alfa, epoetina zeta y filgrastim) (Cuadro 2).

La aprobación de biosimilares de la eritropoyetina alfa (Epoetin alfa $\mathrm{Hexal}$ ) y del filgrastim (Filgrastim ratiopharm, Filgrastim $\mathrm{H}$ exal), ha clarificado el tema de asignar $\mathrm{DCl}$ para biosimilares. Una designación $\mathrm{DCl}$ idéntica"epoetin alfa", "filgrastim", a la de las marcas de referencia, facilita la intercambiabilidad de los medicamentos.

Como ejemplo de su importancia económica, se puede decir que los tres productos biotecnológicos mas importantes en ventas en EUA filgrastim un factor estimulante de colonias de granulocitos humanos (G-CSF) producido por tecnología de DNA recombinante, epoetin alfa una eritropoyetina que estimula la producción de glóbulos rojos, e interferón alfa- $2 b$ un alfa interferón producido por tecnología de DNA recombinante- cuestan aproximadamente US $\$ 15.000, \$ 10.000$, y $\$ 22.000$, respectivamente por paciente y año de acuerdo con la Generic Pharmaceutical Association ${ }^{16}$.

En al gunos países de $A L$ es especialmentegravosa la falta de garantía de intercambiabilidad en los "biosimilares", lo que está originando auténticos problemas en numerosos servicios de salud o equivalentes, al originarse numerosas demandas judiciales cuando se trata de sustituir una marca por un producto multifuente, la mayor parte de las veces con fallo judicial a favor del usuario y resarcimientos de elevados costos que pueden llegar a desequilibrar cajas de seguros modestas.

Empiezan a introducirse en AL directrices 0 borradores legislativos sobremedicamentos biosimilares (Cuadro 3). La experiencia de rigor y calidad en la aprobación de biosimilares en la UE podría servir de modelo en $A L$, y mientras tanto aprovechar los beneficios de la experiencia europea y facilitar la comercialización de los biosimilares aprobados y comercializados en la UE.

Por lo publicado, no hay actualmente regulación en la UE en ningún nivel nacional que permita activamenteque un farmacéutico cambiela prescripción de un médico para un biosimilar. En 19 de 27 Estados miembros de la UE, se permitelegalmentela sustitución por genéricos (bioequivalentes) y solamente en 4 Estados miembros, la substitución es obligatoria.

Sin duda, la futura introducción de los biosimilares en los mercados de AL y en la asistencia sanitaria va a suponer un avance significativo en el uso eficiente de los recursos y una ayuda a la sostenibilidad de los sistemas de salud. 
Cuadro 2. Biosimilares aprobados para comercialización en los países de la UE por la Agencia Europea del Medicamento (EMA) desde el primero aprobado en 2006 hasta 2010.

\begin{tabular}{|c|c|c|c|}
\hline Producto & Compañia & Biosimilares a & Fecha aprobación EMEA \\
\hline $\begin{array}{l}\text { Somatropina (INN) } \\
\text { (human growth hormone) } \\
\text { - Omnitrope } \\
\text { - Valtropin }\end{array}$ & $\begin{array}{l}\text { Sandoz (Novartis) } \\
\text { BioPartners }\end{array}$ & $\begin{array}{l}\text { Genetropin (Pfizer) } \\
\text { Humatrope (Lilly) }\end{array}$ & $\begin{array}{l}\text { Abril } 2006 \\
\text { Abril } 2006\end{array}$ \\
\hline $\begin{array}{l}\text { Epoetina alfa (INN) } \\
\text { (erythropoietin) } \\
\text { - Binocrit } \\
\text { - Epoetin alfa hexal } \\
\text { - Abseamed }\end{array}$ & $\begin{array}{l}\text { Sandoz } \\
\text { Hexal Biotech (Novartis) } \\
\text { M edice Arzneimittel }\end{array}$ & Eprex / Erypo & $\begin{array}{l}\text { Agosto } 2007 \\
\text { Agosto } 2007 \\
\text { Agosto } 2007\end{array}$ \\
\hline $\begin{array}{l}\text { Epoetina zeta (IN N) } \\
\text { (erythropoietin) } \\
\text { - Silapo } \\
\text { - Retacrit }\end{array}$ & $\begin{array}{l}\text { Stada Arzneimittel } \\
\text { Hospira }\end{array}$ & & $\begin{array}{l}\text { Diciembre } 2007 \\
\text { Diciembre } 2007\end{array}$ \\
\hline $\begin{array}{l}\text { Filgrastim (INN) } \\
\text { - Ratiograstim } \\
\text { - Biograstim } \\
\text { - Tevagrastim } \\
\text { - Filgrastim ratiopharm } \\
\text { - Filgrastim Hexal } \\
\text { - Zarzio } \\
\text { - Nivestim }\end{array}$ & $\begin{array}{l}\text { Ratiopharm } \\
\text { CT Arzneimittel } \\
\text { Teva Generics } \\
\text { Ratiopharm } \\
\text { Hexal } \\
\text { Sandoz } \\
\text { Hospira }\end{array}$ & Neupogen / Amgen & $\begin{array}{l}\text { Septiembre } 2008 \\
\text { Septiembre } 2008 \\
\text { Septiembre } 2008 \\
\text { Septiembre } 2008 \\
\text { Febrero } 2009 \\
\text { Febrero } 2009 \\
\text { Junio de } 2010\end{array}$ \\
\hline
\end{tabular}

Cuadro 3. Directrices o borradores legislativos sobre productos medicinales biológicos similares, identificados en países de America Latina.

- Argentina: Borrador de Directriz publicada en julio de 2008 por ANM AT.

Titulo: Registro y modificaciones de productos medicinales biológicos.

- Brasil: Directriz final publicada en 26 de octubre de 2005.

Titulo: RDC № 315, Regulamento Técnico de Registro, Alterações Pós-Registro e Revalidação de Registro dos Produtos Biológicos Terminados, conforme documento anexo e esta Resolução.

- Chile: Subsecretaría de Salud Pública, 31 de Agosto de 2006.

Titulo: Núm. 121, modificación Decreto o 1.876 de 1995, que aprueba el reglamento del Sistema Nacional de Control de Productos farmacéuticos.

- Colombia: Borrador de Directriz, no se sabe la fecha publicación.

Titulo: Licencia para laboratorios fabricantes de productos biológicos.

- México: Borrador de Directriz publicada el 9 de octubre de 2008.

Titulo: Ley general de medicamentos biotecnológicos.

- Panamá: Emitidas el 6 de Septiembre 2007.

Titulo: Guías para el registro de productos biológicos y biotecnológicos.

- Venezuela: Aprobada en 2001.

Título: normativa que regula la condición necesaria de ensayos clínicos para todos los productos biotecnológicos, rechazándose la condición de biosimilar si no somete su propia información clínica. 


\section{La influencia de la industria farmacéutica y su regulación}

Una política del medicamento socialmente eficiente, es un determinante fundamental para la viabilidad y consolidación de los servicios sanitarios públicos; porque el análisis de los propulsores de crecimiento real del gasto sanitario nos informa que el factor explicativo más relevante de la prestación real media es el insumo farmacéutico. Por consiguiente, la sostenibilidad de la financiación del gasto en los sistemas sanitarios va a depender fundamentalmente del crecimiento de la economía en términos dePIB, del modelo de gestión de la prestación farmacéutica y del control de la prescripción ${ }^{17}$.

Sabemos queel incremento desordenado del gasto farmacéutico disipa y desvía ineficientemente recursos - con un alto coste de oportunidadque sacrifica las potenciales mejoras en el capital intelectual, tecnológico y social de los sistemas sanitarios, sin queello parezca revertir sino en un excesivo grado de medicalización en la sociedad; no en incrementos en la salud de las personas y poblaciones, al menos en términos agregados ${ }^{18}$.

Por ello, el rápido e inexorable crecimiento del gasto farmacéutico en los países socialmente avanzados ${ }^{19}$, hace pertinentes las preguntas de $M$ aynard ${ }^{20,21 \text { : }}$

- ¿Cómo puede este crecimiento ser sostenido?

- ¿Cómo pueden los gobiernos hacer una política en medicamentos más coste-efectiva?

- ¿Quécambios habría quellevar a cabo para que el mercado farmacéutico orientara su política industrial y sus preferencias sociales en mejorar los resultados de salud de la población?

- ¿Cómo redefinir todo el sistema de distribución y sus incentivos, para mejorar la calidad y eficiencia en interés de la sociedad y delos consumidores?

Sin duda, el lobby de las "grandes farmacéuticas" (big-pharma) que operan en el sector de salud, ha penetrado profundamenteen el tejido agencial de los sistemas sanitarios; los políticos y autoridades reguladoras, acorde con la teoría de la elección pública, marcan sus actuaciones y preferencias para adquirir o retener el poder y la relación entreindustria y política puedellegar incluso a ser mutuamente confortable (Figura 2).

Las conclusiones globales del UK House of Commons $\mathrm{H}$ ealth Select Committee acerca de la influencia dela industria farmacéutica fueron claras: la influencia de la industria farmacéutica es

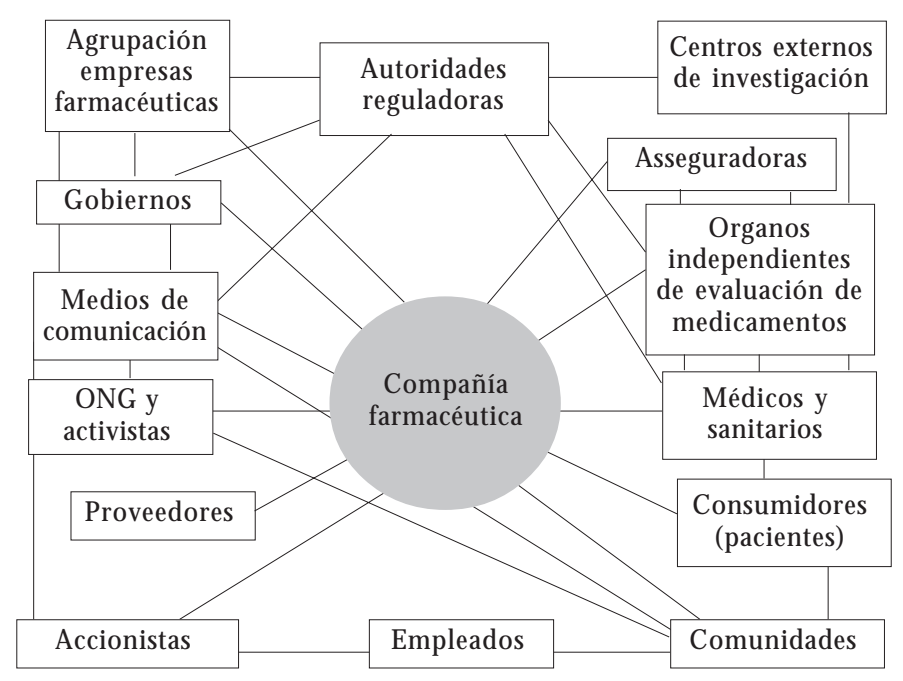

Figura 2. Red de interesados (stakeholders) en la actividad de las compañías farmacéuticas. 
enorme y está fuera de control ${ }^{23}$. El Comitéconsideró que mientras la influencia tradicional delas compañías farmacéuticas se dirigía a los profesionales de la salud, actualmente los tentáculos de las "grandes farmacéuticas" (big-pharma) pe netran y controlan el sector con mucha mayor amplitud, alcanzando a los pacientes y sus organizaciones, departamentos de salud, organismos reguladores, gerentes, investigadores, instituciones benéficas y las Organizaciones no Gubernamentales (ONG), medios académicos y universitarios, medios de comunicación, organizaciones de cuidados de salud, sistema escolar y educativo y partidos políticos ${ }^{24}$.

Algunas consideraciones de dicho Comité24,25 fueron especialmente llamativas: La carencia de chequeos y controles apropiados en el comportamiento de la industria farmacéutica tiene un efecto significativo, perjudicial en la salud delas personas. La industria farmacéutica domina la agenda dela investigación y emprende tal volumen de actividades promocionales hacia prescriptores y consumidores, quelos medicamentos están siendo usados en exceso más allá de lo que es bueno para la salud de las personas. En otro apartado se informa de que la proximidad inadecuada que existe entre la industria y las agencias reguladoras significa que estas fallan en contener las prácticas más dudosas de la industria. Los reguladores parecen inseguros sobresi su prioridad es la seguridad y la eficacia de los medicamentos, o el éxito de las propias compañías farmacéuticas". Las recomendaciones del Comité fueron dirigidas además de al Departamento deSalud del Reino Unido, a los interesados (stakeholders) del sector.

Estos interrogantes han llevado a que desde diferentes ámbitos se estén proponiendo cambios sustantivos en la regulación farmacéutica para promover la competencia frentea la influencia y liberalizar progresivamente determinadas actividades de la cadena de valor en este sector, planteando alternativas de desintermediación y de impulso de la competencia para mejorar la calidad, eficiencia y valor social en los circuitosy canales de distribución; además de fortalecer los mecanismos de participación y corresponsabilidad de los consumidores y usuarios ${ }^{26}$.

Asimismo y bajo prismas diferentes, otros autores ${ }^{27-29}$ han venido profundizando en propuestas y posibles actuaciones correctoras para racionalizar el gasto público en medicamentos, mediante un rediseño integral en la cadena de valor dela prestación farmacéutica, respondiendo a criterios racionales de adecuación, eficiencia y equidad.
Pero también es responsabilidad de las administraciones públicas establecer un marco estable de relaciones con el sector farmacéutico que garantice seguridad, confianza para potenciar de forma sostenida un desarrollo industrial competitivo, una política científica socialmente relevante, un crecimiento delas bases de conocimiento en los servicios sanitarios, una mayor productividad en la inversión y una gestión eficiente delos procesos de innovación.

\section{Crisis de racionalidad \\ en el uso del medicamento \\ y en la investigación biomédica}

La política del medicamento debe sustentarse sobre cuatro ejes principales: (1) Crear incentivos apropiados para potenciar aquella innovación que aporte valor (efectividad y seguridad); (2) Desarrollar instrumentos que garanticen la transparencia social de los procesos biomédicos y minimicen los conflictos deinterés; (3) Primar el juicio técnico independiente en las decisiones sobrequé tratamientos, en qué medida y para quienes la sociedad debe garantizar su apoyo económico; y (4) Impulsar políticas activas para alinear los intereses entre los agentes principales del sector en relación al uso apropiado del medicamento ${ }^{30}$.

La racionalización del gasto farmacéutico exige estrategias para una adecuada utilización de los recursos terapéuticos disponibles; sin embargo, son muchos los factores deinfluencia quedebilitan las políticas públicas tanto para el uso racional demedicamentos como para la gestión y control de los procesos de investigación biomédica.

Sobre este segundo aspecto, sabemos que la industria farmacéutica promueve y controla la mayor parte de la investigación biomédica en el sector. A esterespecto, es llamativo y a la vez preocupante que: (1) Tanto los estudios de metanálisis como los ensayos clínicos financiados por la industria farmacéutica, arrojan claramente conclusiones positivas para sus intereses económi$\cos ^{31,32}$; (2) La probabilidad de que un ensayo clínico financiado por una compañía farmacéutica tenga resultados favorables a la empresa es cuatro veces mayor quesi la fuente definanciación es pública; y no debemos olvidar de que 3 decada 4 ensayos publicados en las principales revistas científicas médicas del mundo están financiados por esta industria; (3) La elaboración de guías clínicas puede no ser objetiva, al no observar con suficienterigor criterios epidemiológicosy evidencias científicamente contrastadas ${ }^{33-35}$; (4) Las re- 
vistas médicas pueden llegar a ser una mera extensión de la estrategia de marketing comercial de las compañías farmacéuticas; (5) Los códigos de buenas prácticas no se cumplen escrupulosamente ${ }^{36} ;$ y (6) La industria farmacéutica puede estar cambiando el comportamiento moral de los médicos. El nexo entrelos viajes a congresosy otros incentivos de la industria farmacéutica a los médicos está estrechamente relacionado con las peticiones e indicaciones prescriptivas de éstos a favor de la compañía financiadora ${ }^{37,38}$.

Por ello, las declaraciones de intenciones (códigos, responsabilidad social corporativa, etc.) no son suficientes para corregir los comportamientos y prácticas inapropiadas de la industria y grupos profesionales adyacentes, que pueden distorsionar la realidad 39,40 .

Para subsanar los conflictos de interés y mejorar los patrones de transparencia en las relaciones agenciales, el Gobierno O bama, ha incorporado en su agenda federal reformista un código de ética universal para contribuir a subsanar la erosionada confianza pública en las relaciones entrela industria y las instituciones representativas de la profesión médica. Ello significa un paso más en el proyecto de ley aprobado en el Congreso deEEUU, que introduceel Physician Paymments Sunshine Act como garantía de transparencia en esta relación ${ }^{41}$.

A pesar de la crisis de innovación disruptiva en el sector biofarmacéutico, los agentes industriales (y toda la amplia cadena de agentes de distribución y dispensación) sehan acostumbrado a extraer beneficios extraordinarios, y cuentan con algunos mecanismos para seguir vendiendo pseudo-innovación, o incluso precipitar lacomercialización demoléculas poniendo en riesgo la seguridad de los pacientes por un uso excesivo e inapropiado. Esta tendencia irracional está claramente estimulada por el marketing comercial y posibilitada por la ausencia o debilidad de información independientey de criterios de planificación, difusión y utilización de las tecnologías e insumos farmacéuticos ${ }^{42}$.

El mayor gasto en farmacia o tecnología no es bueno o malo en sí mismo, pues su adecuación depende de los resultados finales en ganancias de cantidad y calidad devida quese obtengan. La preocupación de muchos radica precisamente en que el crecimiento exponencial queestamos experimentando desde hace varias décadas se combina con un estancamiento de los resultados en salud (Io quellaman la ley delosrendimientosdecrecientes).

La ciencia y la técnica médicas, aunque han tenido notables avances, tienen límites que no pueden traspasarse; hay un choque entre expectativas desmedidas (alimentadas desde el ámbito comercial y profesional) y resultados modestos; dichas expectativas llevan a distorsionar la percepción de los pacientes respecto a su problema de salud, creando la fantasía de que la medicina tiene respuestas para todos los problemas (incluido el envejecimiento) con tal de aplicar suficientes recursos y tecnologías; ésta atribución deomnipotencia, lleva a graves problemas de despilfarro de recursos e ineficiencia asignativa y social en el conjunto de los sistema sanitarios ${ }^{43}$.

La medicalización del malestar nos amenaza como un nuevo problema de salud, como una neurosis de la salud perfecta, de la estética juvenil, y de la prevención irracional einsensata, que viene dela mano deun modelo de "consumismo" médico estéril y alienante ${ }^{44}$.

Por ello, un contrato social renovado implícito entre la industria, administraciones públicas y profesiones debe incluir algunos cambios que modulen la acción de los agentes comerciales (publicidad engañosa), promuevan una educación e información sanitaria adecuadas, controlen los conflictos de interés, incentiven la inversión pública en formación continuada einvestigación biomédicas y fomenten un nuevo profesionalismo sanitario sustentado en valores éticos y sociales.

La responsabilidad social y el buen gobierno en el sector farmacéutico

El proceso deglobalización y su impacto en la economía y en la sociedad, así como las crisis de legitimación pública que ha venido sufriendo la empresa internacional en lasúltimas décadas (Enron, World Com, Bhopal, Nike, Exxon Valdez, etc.), están contribuyendo a una mayor conciencia social en la dinámica de la gobernanza delas compañías, exigiendo mayor democratización y transparencia de la información para los interesados.

No existe una visión universal sobre el compromiso dela empresa en la sociedad, siendo constatable que en los EUA, se da mayor significación al concepto deética dela empresa, queseidentifica con el cumplimiento de la ley y determinadas costumbres del acervo jurídico y de su visión empresarial; sin embargo, en Europa, ha permeabilizado en la cultura empresarial y pública el término de responsabilidad social corporativa (RSC), como consecuencia de la propia arquitectura y del arraigado modelo social paneuropeo.

La rendición de cuentas es un requisito imprescindible para garantizar la transparencia y el 
reconocimiento explícito delos deberes y obligaciones que genera una política social responsable, creando en este proceso de capitalización nuevo patrimonio cívico, cultural y medioambiental como parte del compromiso ético de la empresa con la sociedad y la comunidad a la que sirve.

Para muchas instituciones gubernamentales, buen gobierno integra nuevas modalidades de regulación y un conjunto de instrumentos competenciales que permiten al canzar una mayor eficiencia en la prestación de servicios a la sociedad, favoreciendo la creación de capital social y los derechos de ciudadanía $a^{45-47}$.

Las compañías farmacéuticas son especialmente activas en presentarse a sí mismas como empresas que actúan con principios éticosy sentido de ciudadanía corporativa. En su web corporativa y en sus memorias anuales la responsabilidad social es parte relevante de las mismas.

Las críticas a las firmas farmacéuticas se han exacerbado en los últimos años, al entender que su comportamiento ético-empresarial no se corresponde con los valores corporativos que se insertan en su misión ${ }^{48}$.

La inversión en marketing comercial y variadas modalidades de promoción farmacéutica de las grandes compañías del sector es simplemente orweliana, y cuya repercusión en el precio final de los medicamentos es irrefutable. Algunas de las estrategias de innovación comercial de las "grandes farmacéuticas" (big-pharma) en los actuales patrones de negocio no contribuyen razonablementea la sostenibilidad y equilibrio financiero de los sistemas sanitarios: orientación preferente a los fármacos "yo también" (metoo); dinámicas poco transparentes para prolongar las patentes de los productos "grandes ventas" (blockbuster); desatención por las enfermedades de los países pobres; presión vertical sobrelos agentes del sector y distorsión de la realidad sobre la investigación de resultados coste-efectiva en la práctica clínica.

En la práctica de gobierno de las"grandes farmacéuticas" (big-pharma) se constata la orien- tación al beneficio, la creciente y sostenida valoración en el mercado bursátil, la alta rentabilidad sobre recursos propios e inversión, la exuberante acumulación de capital en las últimas décadas y las llamativas compensaciones económicas a los equipos directivos, vienen a contradecir de alguna manera las afirmaciones que se recogen en sus misiones corporativas ${ }^{49}$. Por más, las llamativas remuneraciones, primas, bonus y otros incentivos económicos de los altos ejecutivos de la industria farmacéutica, extralimitan toda lógica y degradan la cultura ética de estas compañías que operan en un sector social preferente $e^{50}$.

Creemos que el derecho a la salud debe ser primordial por encima delos intereses económicos de las empresas farmacéuticas, y por ello de beríaser pertinente un más justo equilibrio entre innovación y precios, y un régimen de patentes que debería ser revisado para favorecer a los países en desarrollo; con todo ello, la responsabilidad corporativa de estas poderosas compañías quedaría reforzada y su compromiso social estaría legítimamente reconocido por la propia sociedad.

\section{Consideracionesfinales}

La crisis económica y la necesidad de contención del gasto sanitario exige reducir drásticamente la tendencia a originar fuertes incrementosen la factura farmacéutica financiada públicamente, para poder asumir las innovaciones sin comprometer la sostenibilidad de los sistemas de salud. Entre las medidas que pueden ayudar a conseguirlo se pueden señalar: introducir el criterio deeficiencia en la financiación pública denuevosmedicamentos; mejorar la regulación de los medicamentos y de la industria farmacéutica y su cumplimiento; introducir o renovar un contrato social basado en valores éticos y sociales entre la industria, las administraciones públicas y la representación de los profesionales sanitarios; y garantizar el compromiso de la responsabilidad social corporativa en las compañías farmacéuticas. 


\section{Colaboradores}

A Iñesta participó en la concepción teórica, elaboración, redacción final del texto y de la revisión bibliográfica; LA Oteo participó en la concepción teórica, elaboración y redacción final del texto.

\section{Referencias}

1. Bloomberg on Health Care. [site in Internet]. 2009 [cited 2010 M ay 31]. Available from: http://www. bloomberg.com/

2. Global Rank. December. [site in Internet]. 2009 [cited 2010 M ay 31]. Available from: http://www. ft.com/ ft-500-quarterly

3. European Commission. The 2008 EU Industrial R\&D Investment Scoreboard [document in Internet]. 2009 [cited $2010 \mathrm{M}$ ay 31]. Available from: http://iri.jrc. ec.europa.eu/research/docs/2008/pr_es.pdf

4. IM S H ealth 2010. IM S N ational Sales Perspectives [document in Internet]. [cited $2010 \mathrm{M}$ ay 31]. Available from: http://www.imshealth.com/

5. The five myths of generic competition. A Pharma M atters Report. [document in Internet]. 2009 March [cited $2010 \mathrm{M}$ ay 31]. Available from: http:// thomsonreuters.com/content/PDF/scientific/pharma/ generic_myths.pdf

6. Smith $R$. Curbing the influence of the drug industry: a British view. Plos M ed 2005; 2(9):241.

7. IMS Health 2010. IM S H ealth M arket Prognosis [document in Internet]. [cited $2010 \mathrm{M}$ ay 31]. Available from: http://www.imshealth.com/

8. Maynard A, Cookson R. Money or your life? The health-wealth trade-off in pharmaceutical regulation. J Health Serv Res Policy 2001; 6(3):186-189.

9. IM S 2009 Pharmaceutical market forecast. Pharmerging. [site in Internet]. [cited $2010 \mathrm{M}$ ay 31]. Available from: http://www.imshealth.com/

10. Organização Mundial da Saúde (OMS). Geneva: OM S; 2011. [site in Internet]. [cited 2011 May 16]. Available from: http://www.who.int/en/

11. National Center for Pharmacoeconomics. Guidelines for inclusion of drug costs in pharmacoeconomic evaluations [document in Internet]. [cited $2010 \mathrm{M}$ ay 31]. Available from: http://www.ncpe.ie/u_docs/ doc_190.pdf

12. Pharmacoeconomic guidelines around the world. [document in Internet]. [cited $2010 \mathrm{M}$ ay 31]. Available from: http://www.ispor.org/PEguidelines/index.asp

13. Luiza VL, Osorio de Castro C, M arin N, editores. Vigilancia y evaluación de políticas farmacéuticas nacionales. Rio de Janeiro: Organização Pan-Americana da Saúde, Escola Nacional de Saúde Pública Sergio Arouca; 2006.

14. Iñesta GA. Genéricos: medidas para el aumento de su prescripción y uso en el Sistema Nacional de Salud. Madrid: Fundación Alternativas; 2007. Documento de trabajo 123/2007. [site in Internet]. [cited 2010 M ay 31]. Disponible en: http://www.falternativas. org/content/download/5793/165811/version/1/file/ 12c0 08-11-07 doc123.pdf

15. Grupo de Trabajo de BE, Red PARF. Marco para la ejecución de los requisitos de equivalencia para los productos farmacéuticos. Organização Panamericana de Saúde. Noviembre 2008. [site in Internet]. [cited $2010 \mathrm{M}$ ay 31]. Disponible en: http://new. paho.org/hq/index.php?option=com_docman $\&$ task $=$ doc download $\&$ gid $=2207 \& \mid$ temid $=$

16. Generic Pharmaceutical Association. [site in Internet]. [cited 2011 May 16]. Available from: http:// www.gphaonline.org/ 
17. Informe del Consejo Económico y Social de España. [site in Internet]. 2010 [cited $2011 \mathrm{M}$ ay 16]. Available from: http://www.ces.es/index.jsp.

18. Peiró S. M édicos e industria farmacéutica: para estar así, mejor casados. Rev Calidad Asistencial 2009; $24(2): 47-50$.

19. Organisation for Economic Co-operation and Development (OECD). H ealth data 2009 [site in Internet]. [cited 2010 May 31]. Available from: http:// www.ecosante.org/oecd.htm

20. M aynard A. Quality control in the regulation of pharmaceuticals. Pharmaeconomics 2005; 23(5):421-422.

21. M aynard A, Bloor K. Dilemmas in regulation of the market for pharmaceuticals. Health Affairs 2003; 22(3):31-41.

22. M elé $D$. Las cinco mayores compañías farmacéuticas: imagen corporativa y críticas en responsabilidad social. M adrid: IESE Business School; 2006. [cited 2010 $M$ ay 31]. Disponible en: http://www.iese.edu/research/pdfs/OP-06-05.pdf

23. House of Commons Health Committee. The influence of the pharmaceutical industry. Fourth Report of Session 2004-05. M arch 22, 2005. [site in Internet]. [cited $2010 \mathrm{M}$ ay 31]. Available from: http:// www.publications.parliament.uk/pa/cm200405/cmselect/cmhealth/42/42.pdf

24. Collier J. Big pharma and the UK government. The Lancet 2006; 367:97-98.

25. Government response to the Health Committee's Report on the Influence of the Pharmaceutical Industry. September, 2005. [site in Internet]. [cited 2006 Aug 8]. Available from: http://www.dh.gov.uk/ assetRoot/04/11/86/08/04118608.pdf.

26. Meneu R. Regulación y competencia en el sector farmacéutico. En: Repullo JR, Oteo LA, editores. Un nuevo contrato social para un sistema de salud sostenible. Barcelona: Editorial Ariel; 2005.

27. Puig J, Llop J. Propuestas de racionalización y financiación del gasto público en medicamentos. Madrid: Fundación Alternativas; 2004.

28. Villanueva P, Peiró S, Librero J, Pereiro I. Accuracy of pharmaceutical advertisements in medical journals. The Lancet 2003; 361:27-32.

29. López Casasnovas G. Política del medicamento en el contexto de un sistema sanitario multijurisdiccional. En: M eneu R, Peiró S, editores. Elementos para la gestión de la prescripción y la prestación biofarmacéutica. Barcelona: M asson; 2004.

30. Iñesta A. Políticas de medicamentos. En: Repullo JR, Oteo LA, editores. Un nuevo contrato social para un sistema de salud sostenible. Barcelona: Editorial Ariel; 2005

31. Kirsch I, Deacon BJ, Huedo-M edina TB, Scoboria A, Moore TJ, Johnson BT. Initial severity and antidepressant benefits: a meta-analysis of data submitted to the food and drug administration. PLOS $M$ ed 2008; 5(2):45.

32. DeAngelis $C D$, Fontanarosa PB. Impugning the integrity of medical science: the adverse effects of industry influence. JAM A 2008; 299(15):1833-1835.

33. Bossuyt PM, Reitsma JB, Bruns DE, Gatsonis CA, Glasziou PP, Irwig LM, Lijmer JG, M oher D, Rennie D, de Vet HCW. Towards complete and accurate reporting of studies of diagnostic accuracy: the STARD initiative. Ann Intern Med 2003; 138:40-44.
34. Guyatt GH, Rennie D, Editores. U sers' guides to the medical literature: a manual for evidence-based clinical practice. Chicago: American Medical Association; 2002.

35. Von Elm E, Altman DG, Egger M, Pocock SJ, Gotzsche PC, Vandenbroucke JP. STROBE Initiative. The strengthening the reporting of observational studies in epidemiology (STROBE) statement: Guidelines for reporting observational studies. Epidemiology 2007;18:800-804.

36. Smith R. Medical journal are an extension of the marketing arm of pharmaceutical companies. PLOS M ed 2005; 2(5):364-366.

37. Kassirer JP. On the take: how medicine's complicity with big business can endanger your health. New York: Oxford University Press; 2004.

38. Wazana A. Phisicians and the pharmaceutical industry: is a gift ever just a gift? JAM A 2000; 283(3):373-380.

39. Horton R. The dawn of Mc science. New York Rev Books 2004; 51(4):7-9.

40. Oteo LA. La turbidez perpetua en las relaciones de la profesión médica y la industria biofarmacéutica. Rev Calidad Asistencial 2009; 24(4):176-180.

41. Policy and Medicine. Physician payment sunshine provisions: patient protection affordable care act passed the house. [document in Internet]. $2010 \mathrm{M}$ ar 22 [cited 2010 May 31]. Available from: http://www. policymed.com/

42. Peiró $S$, Bernal $E$. ¿A qué incentivos responde la utilización hospitalaria en el Sistema Nacional de Salud? En: Palomo L, Ortún V, García-Benavides F, Márquez-Calderón S, organizadores. Informe SESPAS 2006: los desajustes en la salud en el mundo desarroIlado. Gaceta Sanitaria 2006; 20(Supl.1):110-116.

43. Dana J, Loewenstein G. A social science perspective on gifts to physicians from industry. JAM A 2003; 290(2):252-255.

44. Repullo JR. Presentación del Informe SESPAS, 2006 [editorial]. En: Palomo L, Ortún V, García-Benavides F, Márquez-Calderón $\mathrm{S}$, organizadores. Informe SESPAS 2006: Ios desajustes en la salud en el mundo desarrollado. Gaceta Sanitaria 2006; 20(Supl.1):1.

45. Inkpen A, Tsang E. Social capital, networks, and knowledge transfer. Academy of Management Review 2005; 30(1):146-165.

46. Woolcock M. Social capital and economic development: toward a theoretical synthesis and policy framework. Theory and Society 1998; 27:151-208.

47. Ortún V. El buen gobierno sanitario: economía de la salud y gestión sanitaria. Madrid: Springer Healthcare Communications Ibérica SL; 2009.

48. Angell $M$. The pharmaceutical industry to whom is it accountable? N Engl J Med 2000; 342(25):1902-1904.

49. Angell $M$. The truth about drug companies: how they decisive us an what to do about it. New York: Random House; 2005.

50. Fortune Global 500. FORTUNE Magazine's annual ranking of the world's 500 largest companies. [document in Internet]. [cited $2010 \mathrm{M}$ ay 31]. Available from: http://money.cnn.com/magazines/fortune/ global500/

Artigo apresentado em 14/07/2010

A provado em 25/10/2010

Versão final apresentada em 07/01/2011 